\title{
Saturation of Nuclear Partons: the Fermi Statistics or Nuclear Opacity?
}

\author{
N.N. Nikolaev ${ }^{a, b)}$, W. Schäfer ${ }^{c)}$, B.G. Zakharov ${ }^{b)}$, V.R. Zoller ${ }^{d)}$ \\ a) Institut f. Kernphysik, Forschungszentrum Jülich, D-52425 Jülich, Germany \\ b) L.D.Landau Institute for Theoretical Physics, Chernogolovka, Russia \\ c) NORDITA, Blegdamsvej 17, DK-2100 Copenhagen $\varnothing$, Denmark \\ d) Institute for Theoretical and Experimental Physics, Moscow, Russia \\ E-mail: N.Nikolaev@fz-juelich.de
}

\begin{abstract}
We derive the two-plateau momentum distribution of final state (FS) quarks produced in deep inelastic scattering (DIS) off nuclei in the saturation regime. The diffractive plateau which dominates for small $\mathbf{p}$ measures precisely the momentum distribution of quarks in the beam photon, the rôle of the nucleus is simply to provide an opacity. The plateau for truly inelastic DIS exhibits a substantial nuclear broadening of the FS momentum distribution. We discuss the relationship between the FS quark densities and the properly defined initial state (IS) nuclear quark densities. The Weizsäcker-Williams glue of a nucleus exhibits a substantial nuclear dilution, still soft IS nuclear sea saturates because of the anti-collinear splitting of gluons into sea quarks.
\end{abstract}

The interpretation of nuclear opacity in terms of a fusion and saturation of nuclear partons has been introduced in 1975 [1] way before the QCD parton model: the Lorentz contraction of relativistic nuclei entails a spatial overlap of partons with $x \lesssim x_{A} \approx$ $1 / R_{A} m_{N}$ from different nucleons and the fusion of overlapping partons results in the saturation of parton densities per unit area in the impact parameter space. The pQCD link between nuclear opacity and saturation has been considered by Mueller [2] and the pQCD discussion of fusion of nuclear gluons has been revived by McLerran et al. [3].

The common wisdom is that in DIS the FS interaction effects can be neglected and the observed momentum distribution of struck partons in the FS coincides with the IS density of partons in the probed hadron. Based on the consistent treatment of intranuclear distortions, we derive the two-plateau spectrum of FS quarks. We find a substantial nuclear broadening of inclusive FS spectra and demonstrate that despite this broadening the FS sea parton density exactly equals the IS sea parton density calculated in terms of the WW glue of the nucleus as defined according to [4]. We pay a special attention to an important point that diffractive DIS in which the target nucleus does not break and is retained in the ground state, makes precisely 50 per cent of the total DIS events [5]. We point out that the saturated diffractive plateau measures precisely the momentum distribution of (anti-)quarks in the $q \bar{q}$ Fock state of the photon. In contrast to DIS off nuclei, the fraction of DIS off free nucleons which is diffractive is negligibly small [6], $\eta_{D} \lesssim 6-10 \%$, and there is little room for genuine saturation effects even at 
HERA. We show how the anti-collinear splitting of WW gluons into sea quarks gives rise to nuclear saturation of the sea despite a substantial nuclear dilution of the WW glue.

We base our analysis on the color dipole formulation of DIS [5, 7, 8, 9, 10] and illustrate our ideas on an example of DIS at $x \sim x_{A} \ll 1$ which is dominated by interactions of $q \bar{q}$ states of the photon. The total cross section for interaction of the color dipole $\mathbf{r}$ with the target nucleon equals

$$
\sigma(r)=\alpha_{S}(r) \sigma_{0} \int d^{2} \boldsymbol{\kappa} f(\boldsymbol{\kappa})[1-\exp (i \boldsymbol{\kappa} \mathbf{r})]
$$

where $f(\boldsymbol{\kappa})$ is related to the unintegrated glue of the target nucleon by

$$
f(\boldsymbol{\kappa})=\frac{4 \pi}{N_{c} \sigma_{0}} \cdot \frac{1}{\kappa^{4}} \cdot \frac{\partial G}{\partial \log \kappa^{2}}, \quad \int d^{2} \boldsymbol{\kappa} f(\boldsymbol{\kappa})=1 .
$$

For DIS off a free nucleon target, see figs. 1a-1d, the momentum spectrum of the FS quark prior the hadronization,

$$
\frac{d \sigma_{N}}{d^{2} \mathbf{p} d z}=\frac{\sigma_{0}}{2} \cdot \frac{\alpha_{S}\left(\mathbf{p}^{2}\right)}{(2 \pi)^{2}} \int d^{2} \boldsymbol{\kappa} f(\boldsymbol{\kappa})\left|\left\langle\gamma^{*} \mid \mathbf{p}\right\rangle-\left\langle\gamma^{*} \mid \mathbf{p}-\boldsymbol{\kappa}\right\rangle\right|^{2}
$$

where $\mathbf{p}$ is the transverse momentum, and $z$ the Feynman variable, coincides, upon the $z$ integration, with the conventional IS unintegrated $\mathbf{p}$ distribution of partons in the target. Notice that the target nucleon is color-excited and there is no rapidity gap in the FS.

In DIS off nuclei one must distinguish the three principal processes with distinct unitarity cuts of the forward Compton amplitude (fig. 1g): the coherent diffraction dissociation $(D)$ of the photon (fig. 1h), quasielastic diffraction dissociation (qel) followed by excitation and breakup of the target nucleus (fig. 1i) - in both of them there is no color flow between the photon debris and the nucleus - , and the truly inelastic (in) DIS with color excitation of nucleons of the target nucleus (fig. $1 \mathrm{j}, \mathrm{k}$ ). A useful guidance for isolation of different processes comes from the coupled-channel formalism presented in [11].

We work in the conventional approximation of two t-channel gluons in DIS off free nucleons, figs. 1a-1d, i.e., neglecting the effect of diffractive DIS (figs. 1e,f) on the total cross section on free nucleons, $\eta_{D} \ll 1$. Then the S-matrix of the quark-nucleon scattering must be computed to the second order in the QCD eikonal $\delta(\mathbf{b})$, and the S-matrix for the color dipole-nucleon scattering takes the form

$$
S\left(\mathbf{b}_{+}, \mathbf{b}_{-}\right)=1+2 i\left[\Delta\left(\mathbf{b}_{+}\right)-\Delta\left(\mathbf{b}_{-}\right)\right]-2\left\langle\left[\delta\left(\mathbf{b}_{+}\right)-\delta\left(\mathbf{b}_{-}\right)\right]^{2}\right\rangle_{0}
$$

Here $\langle\ldots\rangle_{0}$ indicates that we must take only the color singlet component of the two-gluon exchange, and we introduced special notation $\Delta(\mathbf{b})=\delta(\mathbf{b})$ for the color-exchange component of the S-matrix. The color dipole cross section equals $\sigma(\mathbf{r})=2 \int d^{2} \mathbf{b}\left\{1-\langle S(\mathbf{b}+\mathbf{r}, \mathbf{b})\rangle_{0}\right\}$, which relates the QCD eikonal to the gluon structure function of the nucleon,

$$
\int d^{2} \mathbf{b}\langle\delta(\mathbf{b}+\mathbf{r}) \delta(\mathbf{b})\rangle_{0}=\frac{1}{8} \alpha_{S}(\mathbf{r}) \sigma_{0} \int d^{2} \boldsymbol{\kappa} f(\boldsymbol{\kappa}) \exp [i \boldsymbol{\kappa} \mathbf{r}]
$$



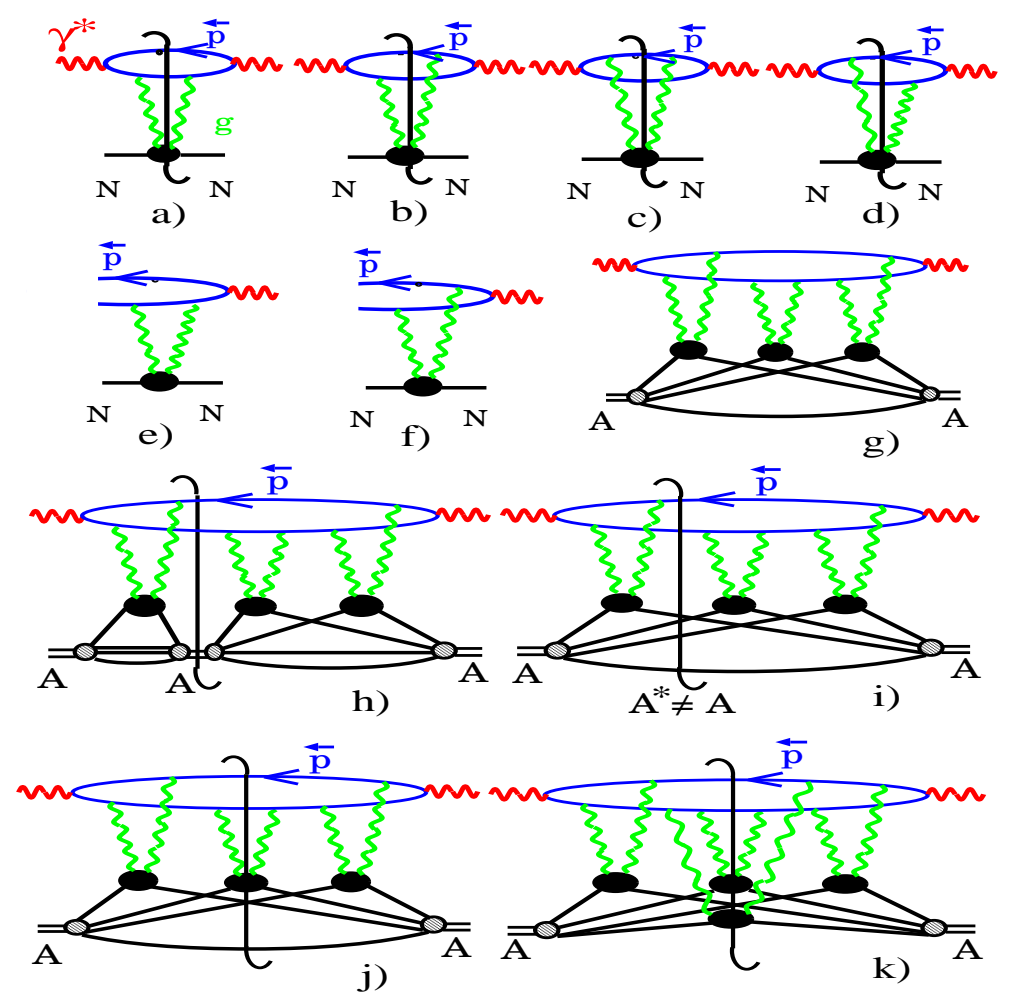

Figure 1: The pQCD diagrams for inclusive (a-d) and diffractive (e,f) DIS off protons and nuclei $(\mathrm{g}-\mathrm{k})$. Diagrams $(\mathrm{a}-\mathrm{d})$ show the unitarity cuts with color excitation of the target nucleon, (g) - a generic multiple scattering diagram for Compton scattering off nucleus, (h) - the unitarity cut for a coherent diffractive DIS, (i) - the unitarity cut for quasielastic diffractive DIS with excitation of the nucleus $A^{*},(\mathrm{j}, \mathrm{k})$ - the unitarity cuts for truly inelastic DIS with single and multiple color excitation of nucleons of the nucleus.

If in the nuclear S-matrix $S_{A}\left(\{\Delta\},\{\delta\} ; \mathbf{b}_{+}, \mathbf{b}_{-}\right)=\prod_{j=1}^{A} S\left(\mathbf{b}_{+}-\mathbf{b}_{j}, \mathbf{b}_{-}-\mathbf{b}_{j}\right)$ one puts $\Delta\left(\mathbf{b}_{ \pm}-\mathbf{b}_{j}\right) \equiv 0$, then it would describe pure diffraction without color excitations in a nucleus. Then, with standard reference to closure [11, the momentum spectrum of observed FS quarks for truly inelastic DIS with color excitations in the nucleus can readily be isolated:

$$
\begin{aligned}
\frac{d \sigma_{i n}}{d z d^{2} \mathbf{p}_{+} d^{2} \mathbf{p}_{-}}= & \frac{1}{(2 \pi)^{4}} \int d^{2} \mathbf{b}_{+}^{\prime} d^{2} \mathbf{b}_{-}^{\prime} d^{2} \mathbf{b}_{+} d^{2} \mathbf{b}_{-} \\
\times & \exp \left[-i \mathbf{p}_{+}\left(\mathbf{b}_{+}-\mathbf{b}_{+}^{\prime}\right)-i \mathbf{p}_{-}\left(\mathbf{b}_{-}-\mathbf{b}_{-}^{\prime}\right)\right] \Psi^{*}\left(\mathbf{b}_{+}^{\prime}-\mathbf{b}_{-}^{\prime}\right) \Psi\left(\mathbf{b}_{+}-\mathbf{b}_{-}\right) \\
\times & \left\{\left\langle A\left|S_{A}^{*}\left(\{\Delta\},\{\delta\} ; \mathbf{b}_{+}^{\prime}, \mathbf{b}_{-}^{\prime}\right) S_{A}\left(\{\Delta\},\{\delta\} ; \mathbf{b}_{+}, \mathbf{b}_{-}\right)\right| A\right\rangle\right. \\
& \left.-\left\langle A\left|S_{A}^{*}\left(0,\{\delta\} ; \mathbf{b}_{+}^{\prime}, \mathbf{b}_{-}^{\prime}\right) S_{A}\left(0,\{\delta\} ; \mathbf{b}_{+}, \mathbf{b}_{-}\right)\right| A\right\rangle\right\}
\end{aligned}
$$

Here $\Psi$ is the $q \bar{q}$-Fock state wave function of the virtual photon, and we suppressed its dependence on $z$. The FS spectra in the coherent and quasielastic diffraction are 
obtained by the substitutions of the expression in the curly braces by

$$
\begin{aligned}
\{\ldots\}_{D}= & \left\langle A\left|1-S_{A}^{*}\left(0,\{\delta\} ; \mathbf{b}_{+}^{\prime}, \mathbf{b}_{-}^{\prime}\right)\right| A\right\rangle\left\langle A\left|1-S_{A}\left(0,\{\delta\} ; \mathbf{b}_{+}, \mathbf{b}_{-}\right)\right| A\right\rangle \\
\{\ldots\}_{q e l}= & \left\{\left\langle A\left|S_{A}^{*}\left(0,\{\delta\} ; \mathbf{b}_{+}^{\prime}, \mathbf{b}_{-}^{\prime}\right) S_{A}\left(0,\{\delta\} ; \mathbf{b}_{+}, \mathbf{b}_{-}\right)\right| A\right\rangle\right. \\
& \left.-\left\langle A\left|S_{A}^{*}\left(0,\{\delta\} ; \mathbf{b}_{+}^{\prime}, \mathbf{b}_{-}^{\prime}\right)\right| A\right\rangle\left\langle A\left|S_{A}\left(0,\{\delta\} ; \mathbf{b}_{+}, \mathbf{b}_{-}\right)\right| A\right\rangle\right\} .
\end{aligned}
$$

The size of color dipoles can be neglected compared to the radius of heavy nuclei. In the standard approximation of a dilute gas nucleus only the color-singlet terms $\propto$ $\left\langle\Delta\left(\mathbf{b}_{1}\right) \Delta\left(\mathbf{b}_{1}\right)\right\rangle_{0}$ would appear in $(\mathbf{6})$, and we find

$$
\begin{gathered}
\left\langle A\left|S_{A}^{*}\left(\{\Delta\},\{\delta\} ; \mathbf{b}_{+}^{\prime}, \mathbf{b}_{-}^{\prime}\right) S_{A}\left(\{\Delta\},\{\delta\} ; \mathbf{b}_{+}, \mathbf{b}_{-}\right)\right| A\right\rangle=\exp \left\{-\frac{1}{2} T(\mathbf{b})\left[\Sigma\left(\mathbf{b}_{+}^{\prime}-\mathbf{b}_{+}\right)\right.\right. \\
\left.\left.+\Sigma\left(\mathbf{b}_{-}^{\prime}-\mathbf{b}_{-}\right)-\Sigma\left(\mathbf{b}_{+}^{\prime}-\mathbf{b}_{-}\right)-\Sigma\left(\mathbf{b}_{+}-\mathbf{b}_{-}^{\prime}\right)+\sigma\left(\mathbf{b}_{+}^{\prime}-\mathbf{b}_{-}^{\prime}\right)+\sigma\left(\mathbf{b}_{+}-\mathbf{b}_{-}\right)\right]\right\},
\end{gathered}
$$

were $T(\mathbf{b})=\int d z n_{A}(z, \mathbf{b})$ is the optical thickness of a nucleus at an impact parameter $\mathbf{b} \approx \mathbf{b}_{ \pm}, \mathbf{b}_{ \pm}^{\prime}$. Here $\Sigma(\mathbf{r})=\sigma(\mathbf{r})$, we use the capital letter just to indicate that it originates from the color excitation processes, whereas the last two terms in the exponent of (9) describe intranuclear attenuation of the $q \bar{q}$ pair due to color-singlet exchanges. The diffractive S-matrix elements entering (7), (8) are readily obtained from (9). For a heavy nucleus the quasielastic diffraction is a surface phenomenon and can be neglected for all the practical purposes, see [5]; it vanishes to the considered leading order (4). If we are interested only in the single particle spectrum and integrate over $\mathbf{p}_{-}$, then $\mathbf{b}_{-}^{\prime}=\mathbf{b}_{-}$and the diffractive attenuation terms in the exponent of (9) would exactly cancel the two terms from multiple color excitation processes in the last line of (9), and

$$
\begin{aligned}
\frac{d \sigma_{i n}}{d^{2} \mathbf{p} d z} & =\frac{1}{(2 \pi)^{2}} \int d^{2} \mathbf{b} \int d^{2} \mathbf{r}^{\prime} d^{2} \mathbf{r} \exp \left[i \mathbf{p}\left(\mathbf{r}^{\prime}-\mathbf{r}\right)\right] \Psi^{*}\left(\mathbf{r}^{\prime}\right) \Psi(\mathbf{r}) \\
& \times\left\{\exp \left[-\frac{1}{2} \sigma\left(\mathbf{r}-\mathbf{r}^{\prime}\right) T(\mathbf{b})\right]-\exp \left[-\frac{1}{2}\left[\sigma(\mathbf{r})+\sigma\left(\mathbf{r}^{\prime}\right)\right] T(\mathbf{b})\right]\right\}
\end{aligned}
$$

Evidently, the dependence of nuclear attenuation factors on $\mathbf{r}, \mathbf{r}^{\prime}$ shall distort strongly the observed momentum distribution of quarks.

In the further interpretation of these results in terms of the parton model we resort to the NSS representation [4]

$$
\Gamma_{A}(\mathbf{b}, \mathbf{r})=1-\exp \left[-\frac{1}{2} \sigma(r) T(\mathbf{b})\right]=\int d^{2} \boldsymbol{\kappa} \phi_{W W}(\boldsymbol{\kappa})[1-\exp (i \boldsymbol{\kappa} \mathbf{r})]
$$

Driven by a close analogy to (11),(2) in terms of $f(\boldsymbol{\kappa})$, we interpret

$$
\phi_{W W}(\boldsymbol{\kappa})=\sum_{j=1}^{\infty} \nu_{A}^{j}(\mathbf{b}) \cdot \frac{1}{j !} f^{(j)}(\boldsymbol{\kappa}) \exp \left[-\nu_{A}(\mathbf{b})\right]
$$

as the Weizsäcker-Williams (WW) unintegrated glue of a nucleus per unit area in the impact parameter plane. It is normalized as

$$
\int d^{2} \boldsymbol{\kappa} \phi_{W W}(\boldsymbol{\kappa})=1-\exp \left[-\nu_{A}(\mathbf{b})\right]
$$


Here

$$
\nu_{A}(\mathbf{b})=\frac{1}{2} \alpha_{S}(r) \sigma_{0} T(\mathbf{b})
$$

defines the nuclear opacity and the $j$-fold convolutions

$$
f^{(j)}(\boldsymbol{\kappa})=\int \prod_{i=1}^{j} d^{2} \boldsymbol{\kappa}_{i} f\left(\boldsymbol{\kappa}_{i}\right) \delta\left(\boldsymbol{\kappa}-\sum_{i=1}^{j} \boldsymbol{\kappa}_{i}\right)
$$

describe the contribution to the diffractive amplitudes from $j$ split pomerons [4].

A discussion of the nuclear antishadowing property of the hard WW glue is found in [4]. A somewhat involved analysis of the properties of the convolutions (14) in the soft region shows that they develop a plateau-like behaviour with the width of the plateau which expands $\propto j$. The gross features of the WW nuclear glue in the soft region are well reproduced by

$$
\phi_{W W}(\boldsymbol{\kappa}) \approx \frac{1}{\pi} \frac{Q_{A}^{2}}{\left(\boldsymbol{\kappa}^{2}+Q_{A}^{2}\right)^{2}},
$$

where the saturation scale $Q_{A}^{2}=\nu_{A}(\mathbf{b}) Q_{0}^{2} \propto A^{1 / 3}$. The soft parameters $Q_{0}^{2}$ and $\sigma_{0}$ are related to the integrated glue of the proton in the soft region,

$$
Q_{0}^{2} \sigma_{0} \sim \frac{2 \pi^{2}}{N_{c}} G_{\text {soft }}, \quad G_{\text {soft }} \sim 1
$$

Notice the nuclear dilution of soft WW glue, $\phi_{W W}(\boldsymbol{\kappa}) \propto 1 / Q_{A}^{2} \propto A^{-1 / 3}$.

On the one hand, making use of the NSS representation, the total nuclear photoabsorption cross section can be cast in the form

$$
\sigma_{A}=\int d^{2} \mathbf{b} \int d z \int \frac{d^{2} \mathbf{p}}{(2 \pi)^{2}} \int d^{2} \boldsymbol{\kappa} \phi_{W W}(\boldsymbol{\kappa})\left|\left\langle\gamma^{*} \mid \mathbf{p}\right\rangle-\left\langle\gamma^{*} \mid \mathbf{p}-\boldsymbol{\kappa}\right\rangle\right|^{2}
$$

which has a profound semblance to (3) and one is tempted to take the differential form of (16) as a definition of the IS sea quark density in a nucleus:

$$
\frac{d \bar{q}_{I S}}{d^{2} \mathbf{b} d^{2} \mathbf{p}}=\frac{1}{2} \cdot \frac{Q^{2}}{4 \pi^{2} \alpha_{e m}} \cdot \frac{d \sigma_{A}}{d^{2} \mathbf{b} d^{2} \mathbf{p}}
$$

In terms of the WW nuclear glue, all intranuclear multiple-scattering diagrams of fig. $1 \mathrm{~g}$ sum up to precisely the same four diagrams fig. 1a-1d as in DIS off free nucleons. Furthermore, one can argue that the small- $x$ evolution of the so-defined IS nuclear sea is similar to that for a free nucleon sea. Although $\mathbf{p}$ emerges here just as a formal Fourier parameter, we shall demonstrate that it can be identified with the momentum of the observed final state antiquark.

On the other hand, making use of the NSS representation, after some algebra one finds

$$
\begin{array}{r}
\exp \left[-\frac{1}{2} \sigma\left(\mathbf{r}-\mathbf{r}^{\prime}\right) T(\mathbf{b})\right]-\exp \left[-\frac{1}{2}\left[\sigma(\mathbf{r})+\sigma\left(\mathbf{r}^{\prime}\right)\right] T(\mathbf{b})\right]=\int d^{2} \boldsymbol{\kappa} \phi_{W W}(\boldsymbol{\kappa}) \\
\left\{\left(\exp \left[i \boldsymbol{\kappa}\left(\mathbf{r}-\mathbf{r}^{\prime}\right)\right]-1\right)+(1-\exp [i \boldsymbol{\kappa} \mathbf{r}])+\left(1-\exp \left[i \boldsymbol{\kappa} \mathbf{r}^{\prime}\right]\right)\right\} \\
-\int d^{2} \boldsymbol{\kappa} \phi_{W W}(\boldsymbol{\kappa})(1-\exp [i \boldsymbol{\kappa} \mathbf{r}]) \int d^{2} \boldsymbol{\kappa}^{\prime} \phi_{W W}\left(\boldsymbol{\kappa}^{\prime}\right)\left(1-\exp \left[i \boldsymbol{\kappa}^{\prime} \mathbf{r}^{\prime}\right]\right)
\end{array}
$$




$$
\begin{aligned}
\frac{d \sigma_{i n}}{d^{2} \mathbf{b} d^{2} \mathbf{p} d z}= & \frac{1}{(2 \pi)^{2}}\left\{\int d^{2} \boldsymbol{\kappa} \phi_{W W}(\boldsymbol{\kappa})\left|\left\langle\gamma^{*} \mid \mathbf{p}\right\rangle-\left\langle\gamma^{*} \mid \mathbf{p}-\boldsymbol{\kappa}\right\rangle\right|^{2}\right. \\
& \left.-\left|\int d^{2} \boldsymbol{\kappa} \phi_{W W}(\boldsymbol{\kappa})\left(\left\langle\gamma^{*} \mid \mathbf{p}\right\rangle-\left\langle\gamma^{*} \mid \mathbf{p}-\boldsymbol{\kappa}\right\rangle\right)\right|^{2}\right\} \\
\frac{d \sigma_{D}}{d^{2} \mathbf{b} d^{2} \mathbf{p} d z}= & \frac{1}{(2 \pi)^{2}}\left|\int d^{2} \boldsymbol{\kappa} \phi_{W W}(\boldsymbol{\kappa})\left(\left\langle\gamma^{*} \mid \mathbf{p}\right\rangle-\left\langle\gamma^{*} \mid \mathbf{p}-\boldsymbol{\kappa}\right\rangle\right)\right|^{2} .
\end{aligned}
$$

As far as diffraction is concerned, the analogy between (20) and its counterpart for free nucleons [4, 8, 12], and nuclear WW glue $\phi_{W W}(\boldsymbol{\kappa})$ and $f(\boldsymbol{\kappa})$ thereof, is complete. Putting the inelastic and diffractive components of the FS quark spectrum together, we evidently find the FS parton density which exactly coincides with the IS parton density (17) such that $\mathbf{p}$ is indeed the transverse momentum of the FS sea quark. The interpretation of this finding is not trivial, though.

Consider first the domain of $\mathbf{p}^{2} \lesssim Q^{2} \lesssim Q_{A}^{2}$ such that the nucleus is opaque for all color dipoles in the photon. Hereafter we assume that the saturation scale $Q_{A}^{2}$ is so large that $\mathbf{p}^{2}, Q^{2}$ are in the pQCD domain and neglect the quark masses. In this regime the nuclear counterparts of the crossing diagrams of figs. 1b,d,f can be neglected. Then, in the classification of [4], diffraction will be dominated by the contribution from the Landau-Pomeranchuk diagram of fig. 1e with the result

$$
\begin{aligned}
\left.\frac{d \bar{q}_{F S}}{d^{2} \mathbf{b} d^{2} \mathbf{p}}\right|_{D} & =\frac{1}{2} \cdot \frac{Q^{2}}{4 \pi^{2} \alpha_{e m}} \cdot \frac{d \sigma_{D}}{d^{2} \mathbf{b} d^{2} \mathbf{p}} \\
& \approx \frac{1}{2} \cdot \frac{Q^{2}}{4 \pi^{2} \alpha_{e m}} \cdot \int d z\left|\int d^{2} \boldsymbol{\kappa} \phi_{W W}(\boldsymbol{\kappa})\right|^{2}\left|\left\langle\gamma^{*} \mid \mathbf{p}\right\rangle\right|^{2} \approx \frac{N_{c}}{4 \pi^{4}}
\end{aligned}
$$

Up to now we specified neither the wave function of the photon nor the spin nor the color representation of charged partons, only the last result in (17) makes explicit use of the conventional spin- $\frac{1}{2}$ partons. We also used the normalization (13). Remarkably, diffractive DIS measures the momentum distribution of quarks and antiquarks in the $q \bar{q}$ Fock state of the photon. We emphasize that this result, typical of the LandauPomeranchuk mechanism, is a completely generic one and would hold for any beam particle such that its coupling to colored partons is weak. In contrast to diffraction off free nucleons [8, 12, 13], diffraction off opaque nuclei is dominated by the anti-collinear splitting of hard gluons into soft sea quarks, $\boldsymbol{\kappa}^{2} \gg \mathbf{p}^{2}$. Precisely for this reason one finds the saturated FS quark density, because the nuclear dilution of the WW glue is compensated for by the expanding plateau. The result (21) has no counterpart in DIS off free nucleons because diffractive DIS off free nucleons is negigibly small even at HERA, $\eta_{D} \lesssim 6-10 \%$.

The related analysis of the FS quark density for truly inelastic DIS in the same domain of $\mathbf{p}^{2} \lesssim Q^{2} \lesssim Q_{A}^{2}$ gives

$$
\begin{aligned}
\left.\frac{d \bar{q}_{F S}}{d^{2} \mathbf{b} d^{2} \mathbf{p}}\right|_{i n} & =\frac{1}{2} \cdot \frac{Q^{2}}{4 \pi^{2} \alpha_{e m}} \cdot \int d z \int d^{2} \boldsymbol{\kappa} \phi_{W W}(\boldsymbol{\kappa})\left|\left\langle\gamma^{*} \mid \mathbf{p}-\boldsymbol{\kappa}\right\rangle\right|^{2} \\
& =\frac{Q^{2}}{8 \pi^{2} \alpha_{e m}} \phi_{W W}(0) \int^{Q^{2}} d^{2} \boldsymbol{\kappa} \int d z\left|\left\langle\gamma^{*} \mid \boldsymbol{\kappa}\right\rangle\right|^{2}=\frac{N_{c}}{4 \pi^{4}} \cdot \frac{Q^{2}}{Q_{A}^{2}} \cdot \theta\left(Q_{A}^{2}-\mathbf{p}^{2}\right)
\end{aligned}
$$


It describes final states with color excitation of a nucleus, but as a function of the photon wave function and nuclear WW gluon distribution it is completely different from (3) for free nucleons. The $\theta$-function simply indicates that the plateau for inelastic DIS extends up to $\mathbf{p}^{2} \lesssim Q_{A}^{2}$. For $Q^{2} \ll Q_{A}^{2}$ the inelastic plateau contributes little to the transverse momentum distribution of soft quarks, $\mathbf{p}^{2} \lesssim Q^{2}$, but the inelastic plateau extends way beyond $Q^{2}$ and its integral contribution to the spectrum of FS quarks is exactly equal to that from diffractive DIS. Such a two-plateau structure of the FS quark spectrum is a new finding and has not been considered before.

Now notice, that in the opacity regime the diffractive FS parton density coincides with the contribution $\propto\left|\left\langle\gamma^{*} \mid \mathbf{p}\right\rangle\right|^{2}$ to the IS sea parton density from the spectator diagram 1a, whereas the FS parton density for truly inelastic DIS coincides with the contribution to IS sea partons from the diagram of fig. 1c. The contribution from the crossing diagrams $1 \mathrm{~b}, \mathrm{~d}$ is negigibly small.

Our results (21) and (22), especially nuclear broadening and unusually strong $Q^{2}$ dependence of the FS/IS parton density from truly inelastic DIS, demonstrate clearly a distinction between diffractive and inelastic DIS. Our considerations can readily be extended to the spectrum of soft quarks, $\mathbf{p}^{2} \lesssim Q_{A}^{2}$, in hard photons, $Q^{2} \gtrsim Q_{A}^{2}$. In this case the result (21) for diffractive DIS is retained, whereas in the numerator of the result (22) for truly inelastic DIS one must substitute $Q^{2} \rightarrow Q_{A}^{2}$, so that in this case $\left.\left.d q_{F S}\right|_{D} \approx d q_{F S}\right|_{\text {in }}$ and $\left.d q_{I S} \approx 2 d q_{F S}\right|_{D}$. The evolution of soft nuclear sea, $\mathbf{p}^{2} \lesssim Q_{A}^{2}$, is entirely driven by an anti-collinear splitting of the NSS-defined WW nuclear glue into the sea partons.

The early discussion of the FS quark density in the saturation regime is due to Mueller [14]. Mueller focused on $Q^{2} \gg Q_{A}^{2}$ and discussed neither a distinction between diffractive and truly inelastic DIS nor a $Q^{2}$ dependence and broadening (15) for truly inelastic DIS at $Q^{2} \lesssim Q_{A}^{2}$.

We come to a summary. We reported a derivation of the FS parton density. Our result (9) summarizes in an elegant way intranuclear distortions due to multiple diffractive rescatterings and color excitations of the target nucleus. In conjunction with the NSS definition of the WW glue of the nucleus, eqs. (11), (12), it gives an explicit form of the FS parton densities. The two-plateau FS quark density with the strong $Q^{2}$ dependence of the plateau for truly inelastic DIS has not been discussed before. A comparison with the IS nuclear parton densities which evolve from the NSS-defined WW nuclear glue shows an exact equality of the FS and IS parton densities. The plateau-like saturated nuclear quark density is suggestive of the Fermi statistics, but our principal point that for any projectile which interacts weakly with colored partons the saturated density measures the momentum distribution in the $q \bar{q}, g g, \ldots$ Fock state of the projectile disproves the Fermi-statistics interpretation. The spin and color multiplet of colored partons the photon couples to is completely irrelevant, what only counts is an opacity of heavy nuclei. The anti-collinear splitting of WW nuclear glue into soft sea partons is a noteworthy feature of the both diffractive DIS and IS sea parton distributions. The emergence of a saturated density of IS sea partons from the nuclear-diluted WW glue is due to the nuclear broadening of the plateau (15). Because the predominance of diffraction is a very special feature of DIS [5], one must be careful with applying the IS parton densities 
to, for instance, nuclear collisions, in which diffraction wouldn't be of any significance.

One can go one step further and consider interactions with the opaque nucleus of the $q \bar{q} g$ Fock states of the photon. Then the above analysis can be extended to $x \ll x_{A}$ and the issue of the $x$-dependence of the saturation scale $Q_{A}^{2}$ can be addressed following the discussion in [9]. We only mention here that as far as diffraction and IS parton densities are concerned, the NSS-defined WW glue remains a useful concept and the close correspondence between $\phi_{W W}(\boldsymbol{\kappa})$ for the nucleus and $f(\boldsymbol{\kappa})$ for the nucleon is retained.

This work has been partly supported by the INTAS grants 97-30494 \& 00-00366 and the DFG grant 436RUS17/119/01, and by DAAD and Nordita.

\section{References}

[1] N.N. Nikolaev and V.I. Zakharov, Sov. J. Nucl. Phys. 21 (1975) 227; [Yad. Fiz. 21 (1975) 434]; Phys. Lett. B55 (1975) 397.

[2] A.H. Mueller, Nucl. Phys. B335 (1990) 115.

[3] L. McLerran and R. Venugopalan, Phys. Rev. D49 (1994) 2233; D55 (1997) 5414;

E. Iancu, A. Leonidov and L. McLerran, Lectures at the Cargèse Summer School, August 6-18, 2001, arXiv:hep-ph/0202270.

[4] N.N. Nikolaev, W. Schäfer and G. Schwiete, JETP Lett. 72 (2000) 583; [Pisma Zh. Eksp. Teor. Fiz. 72 (2000) 583]; Phys. Rev. D63 (2001) 014020.

[5] N.N. Nikolaev, B.G. Zakharov and V.R. Zoller, Z. Phys. A351 (1995) 435.

[6] M. Genovese, N.N. Nikolaev and B.G. Zakharov, J. Exp. Theor. Phys. 81 (1995) 633; [Zh. Eksp. Teor. Fiz. 108 (1995) 1155]

[7] N.N. Nikolaev and B.G. Zakharov, Z. Phys. C49 (1991) 607

[8] N.N. Nikolaev and B.G. Zakharov, Z. Phys. C53 (1992) 331.

[9] N.N.Nikolaev and B.G.Zakharov, J. Exp. Theor. Phys. 78 (1994) 806; [Zh. Eksp. Teor. Fiz. 105 (1994) 1498]; Z. Phys. C64 (1994) 631.

[10] N.N. Nikolaev, B.G. Zakharov and V.R. Zoller, JETP Lett. 59 (1994) 6

[11] N.N. Nikolaev, J. Exp. Theor. Phys. 54 (1981) 434.

[12] N.N. Nikolaev and B.G. Zakharov, Phys. Lett. B332 (1994) 177

[13] M. Genovese, N.N. Nikolaev and B.G. Zakharov, Phys. Lett. B378 (1996) 347

[14] A.H. Mueller, Nucl. Phys. B558 (1999) 285; Lectures at the Cargèse Summer School, August 6-18, 2001, arXiv:hep-ph/0111244. 